\title{
Impact of Appropriate Use on the Estimated Radiation Risk to Men and Women Undergoing Radionuclide Myocardial Perfusion Imaging
}

\author{
Rami Doukky ${ }^{1,2}$, Nathan Frogge ${ }^{2,3}$, Andrew Appis ${ }^{2}$, Kathleen Hayes ${ }^{2}$, George Khoudary ${ }^{2}$, Louis Fogg ${ }^{4}$, and \\ Kim A. Williams, Sr. ${ }^{2}$ \\ ${ }^{1}$ Division of Cardiology, John H. Stroger, Jr. Hospital of Cook County, Chicago, Illinois; ${ }^{2}$ Division of Cardiology, Rush University \\ Medical Center, Chicago, Illinois; ${ }^{3}$ Department of Internal Medicine, University of Chicago Medicine, Chicago, Illinois; and \\ ${ }^{4}$ College of Nursing, Rush University, Chicago, Illinois
}

The impact of appropriate use criteria (AUC) for myocardial perfusion imaging (MPI) with SPECT on the estimated lifetime attributable risk (LAR) of cancer is unknown. Methods: A cohort of 1,511 consecutive patients who underwent clinically indicated ${ }^{99 \mathrm{mTC}} \mathrm{T}$-setamibi MPI were categorized into appropriate/uncertain $(n=823)$ versus inappropriate $(n=688)$ use groups according to the 2009 AUC and were prospectively followed for $27 \pm 10$ mo. Logistic regression models were used to determine the annualized probability of major adverse cardiac events (MACE) of cardiac death or myocardial infarction and the probability of revascularization within 6 mo of MPI, accounting for relevant covariates. We determined LAR for each subject on the basis of accepted risk estimates. We calculated MPI's benefit-torisk ratios, defined by the annualized predicted MACE-to-LAR ratio and the predicted 6-mo-revascularization-to-LAR ratio. Results: During follow-up, there were 22 MACE and 29 6-mo revascularizations. The administered radioactivity and effective radiation doses absorbed were similar between the study groups. Patients with inappropriate MPI had significantly higher LAR (median, $0.08 \%$ vs. $0.06 \%, P<0.001$ ), lower predicted MACE-to-LAR ratio (median, 1.5 vs. $4.3, P<0.001$ ), and lower predicted 6 -mo-revascularizationto-LAR ratio (median, 5.4 vs. $15.5, P<0.001$ ). Women had higher LAR (median, $0.08 \%$ vs. $0.07 \%, P<0.001$ ) and lower predicted MACE-to-LAR ratio (median, 1.9 vs. $3.3, P<0.001$ ) and 6-mo-revascularization-to-LAR ratio (median, 4.4 vs. $17.5, P<$ 0.001). However, appropriate/uncertain use negated the difference between men and women in LAR $(P=0.94)$ and the predicted MACE-to-LAR ratio $(P=0.97)$. Conclusion: Inappropriate MPI use is associated with excess cancer risk and lower MPI's benefit-to-risk ratio. Appropriate/uncertain use neutralizes the sex gap in LAR with MPI.

Key Words: SPECT; myocardial perfusion imaging (MPI); appropriate use criteria (AUC); lifetime attributable risk (LAR); cancer; outcome

J Nucl Med 2016; 57:1251-1257

DOI: 10.2967/jnumed.115.167205

Received Sep. 21, 2015; revision accepted Mar. 14, 2016.

For correspondence or reprints contact: Rami Doukky, John H. Stroger, Jr. Hospital of Cook County, Division of Cardiology, 1901 W. Harrison St., Chicago, IL 60612.

E-mail: rami_doukky@rush.edu

Published online Apr. 21, 2016.

COPYRIGHT (C 2016 by the Society of Nuclear Medicine and Molecular Imaging, Inc.
$\mathbf{M}$ yocardial perfusion imaging (MPI) with SPECT is a pivotal tool in the risk assessment and clinical decision making in patients with known or suspected coronary artery disease (CAD) (1). The costly expansion in MPI use prompted professional organizations to develop appropriate use criteria (AUC) to guide physicians on the optimal use of SPECT MPI (2).

In recent years, there have been growing concerns in regards to the health risks from exposure to low levels of ionizing radiation related to cardiac imaging, particularly MPI (3-7). Although AUC have been developed primarily to curb the excessive spending of health care dollars, using MPI according to AUC has been considered to be an opportunity to reduce patients' exposure to ionizing radiation (8). This is important because multiple investigators demonstrated that patients referred for inappropriate SPECT MPI are often women and younger individuals $(9,10)$, who are at greater lifetime attributable risk (LAR) of cancer (11). The impact of appropriate use on the LAR and the MPI benefit-to-risk ratio, particularly as it relates to patient's sex, has never been examined. A recent scientific statement from the American Heart Association outlined approaches to enhancing radiation safety in cardiovascular imaging. The statement recommended shared decision making, through which patients are made aware of the clinical justification (appropriateness) and expected benefit and potential risks of the test, including radiation-related risk. The statement gives this recommendation a class I designation (beneficial) with level of evidence $\mathrm{C}$ (expert consensus), which underlines lack of data in this domain (8).

In this investigation, we sought to determine the impact of AUC of SPECT MPI on the LAR of cancer and on the benefit-to-risk ratio of such testing in men and women. We hypothesized that inappropriate use would be associated with higher LAR and lower benefit-to-risk ratio. Furthermore, appropriate use would close the sex gap in radiation risk by excluding younger, low-risk women from testing.

\section{MATERIALS AND METHODS}

\section{Patients}

This is a substudy of a previously published multisite prospective cohort of 1,511 consecutive patients referred for outpatient, clinically indicated SPECT MPI performed in a community setting between August 15, 2007, and May 15, 2010. Subjects were enrolled from the offices of 22 physicians from 11 cardiology and primary care practices encompassing 12 zip codes within the Chicago area. The methods applied in the formation and follow-up of this cohort were 
reported elsewhere (9). The study was approved by the institutional review board of Rush University Medical Center.

Briefly, clinical history and rationale for testing were determined at the time of testing, which were then used to calculate Framingham 10-y global coronary heart disease risk (12) and the pretest likelihood of obstructive CAD based on age, sex, and symptom quality (13). A computer-based algorithm written in Microsoft Excel ${ }^{\circledR}$ was applied to categorize each MPI study as appropriate, uncertain, or inappropriate according to the 2009 AUC $(2,9)$. In a hierarchical order, each subject was allocated to 1 of 5 clinical scenarios: preoperative assessment, prior revascularizations, prior imaging, ischemic equivalent, and asymptomatic. Within each hierarchical scenario, as described in the 2009 AUC, we determined MPI appropriateness on the basis of surgical risk, prior revascularization type and date, imaging findings and date, pretest CAD likelihood, ability to exercise, electrocardiogram interpretability, and Framingham 10-y coronary heart disease risk (2). To study the impact of inappropriate use on outcome and LAR, and in alignment with the American Heart Association scientific statement (8), patients with appropriate and uncertain appropriateness scans were combined, stratifying the study cohort into appropriate/uncertain and inappropriate use groups.

\section{Stress MPI}

A 1-day rest-stress ${ }^{99 \mathrm{~m}} \mathrm{Tc}$-sestamibi protocol was implemented (14). An exercise or pharmacologic stress modality was chosen as clinically appropriate (15). MPI studies were acquired using a standard dual-detector, cardiac SPECT camera. All MPI scans were semiquantitatively interpreted by an expert nuclear cardiologist who was masked to clinical and outcome data (9). Using standard methods (9), we quantified the burden of fixed and reversible perfusion abnormalities (infarction plus ischemia) and reversible perfusion abnormality (ischemia) as summed stress score and summed difference score, respectively. A post-stress left ventricular ejection fraction of $50 \%$ or more was considered normal. The transient ischemic dilation ratio, a marker for high-risk scan, was tabulated (16).

\section{Outcome Determination}

Subjects were prospectively followed for a mean of $27 \pm 10$ mo for events of cardiac death, nonfatal myocardial infarction (MI), and surgical or percutaneous coronary revascularization. Outcome assessors were masked to MPI findings and AUC classification. Four methods for ascertaining outcome events were uniformly applied: review of patient health records, mailed questionnaires, telephone interviews, and Social Security Death Index and death certificates. The cardiac outcomes of interest were major adverse cardiac events (MACE), defined as a composite of cardiac death or MI, and coronary revascularization within 6 mo from MPI, as these were considered to be directly triggered by MPI findings.

\section{Determination of Predicted Probability of Outcome Events}

Two multivariable logistic regression models were fit to calculate the predicted probability of MACE (model 1) or revascularization within 6 mo (model 2) as determined by established clinical and imaging predictors (independent variables) known to affect these outcomes $(1,9,12,16)$. The calculated probabilities of MACE were then annualized. The clinical covariates used in calculating the probability of MACE and 6-mo revascularization included age, sex, symptomatic presentation, coronary risk factors (diabetes, hypertension, dyslipidemia, tobacco use, and family history), known CAD, and use of cardioprotective medications (statins, $\beta$-blockers, angiotensin-converting enzyme inhibitors or angiotensin receptor blockers, and antiplatelet agents). The imaging covariates used in calculating the probability of MACE were summed stress score, ejection fraction, and transient ischemic dilation ratio. The imaging covariate used in calculating the probability of 6-mo revascularization was the summed difference score (ischemic burden).
We used the Spearman correlation method to ensure lack of multicollinearity between covariates included in each model. The models were internally validated by conducting 1,000-iteration bootstrap testing. To confirm the accuracy of the selected clinical and imaging predictors in forecasting the observed events, we used receiver-operating-characteristic curves to determine the C-statistic (area under the curve) with 95\% confidence interval (CI) associated with the predicted probabilities. The goodness-of-fit of these models was confirmed using the Hosmer and Lemeshow test.

\section{Effective Radiation Dose and LAR of Cancer}

The sum of organ-specific effective dose was calculated by multiplying the administered ${ }^{99 \mathrm{~m}} \mathrm{Tc}$-sestamibi activity $(\mathrm{MBq})$ received by a conversion factor, as specified in the Radiation Dose Assessment Resource tables and manufacturer's package insert $(17,18)$. Using data provided in Table 12D-1 in the BEIR-VII report (11), we plotted the LAR predicted from a $100 \mathrm{mSv}$ effective dose received by men and women at age 20, 30, 40, 50, 60, 70, and 80, as shown in Supplemental Figure 1 (supplemental materials are available at http://jnm.snmjournals.org) (4). Using the coordinates of the resultant plots, we determined the LAR from $100 \mathrm{mSv}$ of absorbed radiation dose for a given age and sex. For patients older than $80 \mathrm{y}$, the LAR was extrapolated by extending the plotted curves, assuming the same decline in risk observed between ages 70 and $80 \mathrm{y}$ (Supplemental Fig. 1). We chose not to exclude patients older than 80 y because LAR declines with age but is not known to go down to zero. Subsequently, the estimated LAR based on an effective dose of $100 \mathrm{mSv}$ was linearly scaled back according to the actual effective dose received by each patient.

\section{Endpoints}

Because MPI is typically performed for 2 main reasons-stratifying risk and identifying candidates for coronary revascularization-we considered the annualized probability of MACE and the probability of 6-mo revascularization as benefits of MPI, whereas the LAR of cancer was the risk. As a diagnostic test, MPI can identify only candidates for coronary angiography and revascularization; thus, we considered coronary revascularization a benefit of diagnostic testing. Whether subsequent coronary revascularization is appropriate and clinically beneficial to the patient is beyond the scope of this investigation. The primary outcome of the study was the estimated LAR of cancer from a single MPI exposure. The secondary outcomes were clinical benefit-to-radiation risk ratios: the annualized probability of MACE-to-LAR and the probability of 6-mo-revascularization-to-LAR.

\section{Statistical Methods}

Considering that the expected effective dose received from a single MPI is $12 \mathrm{mSv}$, which is associated with a mean LAR of $0.1 \%$, with an SD of $0.14 \%$ (19), we determined that the available sample of 1,511 subjects (45.5\% inappropriate) would provide the study $98 \%$ power to detect $20 \%$ difference in mean LAR between the 2 study groups with a 2-tailed $\alpha$ of 0.05 .

The $\chi^{2}$ or Fisher exact test was used, as appropriate, to compare dichotomous variables, which were expressed as frequencies (percentages). The 2-tailed Student $t$ test and the Mann-Whitney test were used to compare normally distributed (mean $\pm \mathrm{SD}$ ) and skewed (median, interquartile range) continuous variables, respectively. Two-tailed $P$ values of less than 0.05 were considered statistically significant. PASW 18.0 software (SPSS, Inc.) was used for statistical analyses.

In supplemental Monte Carlo analyses (Oracle Crystal Ball, version 11.1.2.3.500, and Excel 2010), we calculated a probability distribution for MACE, 6-mo revascularization, and LAR. We then conducted 10,000 random independent samplings to estimate the lifetime cancer risk from a single MPI exposure. Additionally, 10,000 paired samplings of LAR and MACE or 6-mo revascularization were conducted to estimate MACE-to-LAR and 6-mo-revascularization-to-LAR ratios. 


\section{Sensitivity Analyses}

Because cardiovascular disease may shorten life expectancy, it may be associated with lower LAR and greater MACE and revascularization risks (20). Therefore, we repeated all study analyses in the subset of patients with normal ejection fraction and no known CAD. We also repeated the main study analyses after patients older than $80 \mathrm{y}$ were excluded.

\section{RESULTS}

MPI referrals were appropriate in 779 (51.6\%), inappropriate in $688(45.5 \%)$, and uncertain in 44 (2.9\%) studies, yielding 2 study groups: appropriate/uncertain $(n=823$ [54.5\%]) and inappropriate $(n=688[45.5 \%])$. The baseline clinical and imaging characteristics of the study subjects were previously published and are summarized in Supplemental Table 1 (9). Notably, patients in the inappropriate group were younger and predominantly women; they also had lower prevalence of CAD and coronary risk factors and were less likely to have an abnormal MPI finding.

\section{Outcomes}

During follow-up, 22 (1.5\%) MACE (cardiac death or MI) and 29 (1.9\%) 6-mo revascularizations were observed (Table 1). Detailed outcome events were previously published and are summarized in Supplemental Table 2 (9). The appropriate/uncertain group had a higher MACE rate, whereas the difference in the rate of coronary revascularization within 6 mo was not statistically significant.
Using multivariable logistic regression analysis, we determined the probability of the primary endpoint of MACE for each subject on the basis of clinical and imaging predictors known to affect these outcomes. The C-statistic for model 1 was 0.90 (CI $=0.84-0.96$; $P<0.001)$. The calculated predicted probabilities of MACE for the entire follow-up (mean, $27 \mathrm{mo}$ ) were then annualized. Using multivariable logistic regression analysis, we determined the probability of the revascularization within $6 \mathrm{mo}$. The C-statistic for model 2 was $0.90(\mathrm{CI}=0.84-0.95 ; P<0.001)$. Both models were internally validated by demonstrating that the odds ratios for each covariate fell well within the $95 \%$ CIs from bootstrap testing. The goodnessof-fit for both models was acceptable (Hosmer and Lemeshow $P$ values: model $1=0.55$, model $2=0.77$ ). The means of the predicted probabilities for MACE and 6-mo revascularization were significantly higher in the appropriate/uncertain use group (Table 1).

\section{Radiation Exposure and LAR of Cancer}

As shown in Table 1, the means of ${ }^{99 \mathrm{~m}} \mathrm{Tc}$ radioactivity administered and the effective radiation dose received were not statistically different between the study groups. However, the median LAR was significantly higher in the inappropriate use group (median, 0.08 vs. $0.06 \% ; P<0.001$ ), as shown in Figure 1. Furthermore, the ratio of probability of MACE-to-LAR was significantly lower in the inappropriate versus appropriate/uncertain use group (median, 1.5 vs. $4.3, P<0.001)$. Similarly, the ratio of the probability of 6-mo-revascularization-to-LAR was significantly lower in the

TABLE 1

Outcomes According to Appropriate Use Group

\begin{tabular}{|c|c|c|c|c|}
\hline Variable & $\begin{array}{l}\text { Entire cohort } \\
(n=1,511)\end{array}$ & $\begin{array}{l}\text { Appropriate/uncertain } \\
(n=823 ; 54.5 \%)\end{array}$ & $\begin{array}{c}\text { Inappropriate } \\
(n=688 ; 45.5 \%)\end{array}$ & $P$ \\
\hline \multicolumn{5}{|l|}{ Outcome events $(n)$} \\
\hline MACE (cardiac death or MI) & $22(1.5 \%)$ & $20(2.4 \%)$ & $2(0.3 \%)$ & $<0.001^{*}$ \\
\hline Cardiac death & $12(0.8 \%)$ & $12(1.5 \%)$ & $0(0 \%)$ & $0.001^{*}$ \\
\hline Myocardial infarction & $11(0.7 \%)$ & $9(1.1 \%)$ & $2(0.3 \%)$ & $0.08^{\star}$ \\
\hline REV within 6 mo & $29(1.9 \%)$ & $19(2.3 \%)$ & $10(1.5 \%)$ & $0.23^{\dagger}$ \\
\hline \multicolumn{5}{|c|}{ Median predicted probabilities of events (\%) } \\
\hline MACE: cardiac death or MI & $0.41(0.1-1.13)$ & $0.59(0.24-1.55)$ & $0.27(0.14-0.77)$ & $<0.001^{\ddagger}$ \\
\hline Annualized MACE* & $0.18(0.08-0.50)$ & $0.26(0.10-0.69)$ & $0.12(0.06-0.34)$ & $<0.001^{\ddagger}$ \\
\hline 6-mo REV† & $0.60(0.27-1.49)$ & $0.82(0.34-1.99)$ & $0.44(0.21-1.10)$ & $<0.001^{\ddagger}$ \\
\hline \multicolumn{5}{|l|}{ Median radiation exposure and LAR } \\
\hline Rest ${ }^{99 m} \mathrm{Tc}$ dose (MBq) & $374(363-389)$ & $374(363-389)$ & 374 (363-389) & $0.77^{\ddagger}$ \\
\hline Stress ${ }^{99 m T c}$ dose (MBq) & $1,280(1,228-1,336)$ & $1,277(1,228-1,328)$ & $1,284(1,232-1,343)$ & $0.21^{\ddagger}$ \\
\hline Total $99 \mathrm{~m} T \mathrm{~T}$ dose $(\mathrm{MBq})$ & $1,654(1,598-1,721)$ & $1,654(1,595-1,717)$ & $1,658(1,598-1,728)$ & $0.39 \ddagger$ \\
\hline Effective dose (mSv) & $13.5(13.1-14.0)$ & $13.5(13.1-14.0)$ & $13.5(13.1-14.1)$ & $0.52^{\ddagger}$ \\
\hline LAR of cancer (\%) & $0.07(0.05-0.09)$ & $0.06(0.04-0.08)$ & $0.08(0.07-0.10)$ & $<0.001^{\ddagger}$ \\
\hline \multicolumn{5}{|l|}{ Median benefit-to-risk ratio } \\
\hline Predicted MACE-to-LAR ratio & $2.6(0.9-9.0)$ & $4.3(1.6-15.4)$ & $1.5(0.7-4.6)$ & $<0.001^{\ddagger}$ \\
\hline 6-mo-REV-to-LAR ratio & $9.84(3.6-27.2)$ & $15.5(5.7-39.1)$ & $5.4(2.5-14.5)$ & $<0.001^{\ddagger}$ \\
\hline \multicolumn{5}{|c|}{$\begin{array}{l}{ }^{*} \text { Fisher exact test. } \\
{ }^{\dagger} \chi^{2} \text { test. } \\
{ }^{ \pm} \text {Mann-Whitney test. } \\
\text { REV = revascularization. } \\
\text { Data in parentheses are interquartile ranges unless otherwise indicated. }\end{array}$} \\
\hline
\end{tabular}


inappropriate use group (median, 5.4 vs. $15.5 ; P<0.001$ ), as shown in Figure 1. Therefore, a greater proportion of patients in the inappropriate use group had a predicted annual MACE risk that was less than their LAR (ratio < $<$ ) as compared with the appropriate/ uncertain use group (266 [38.7\%] vs. 144 [17.5\%]; $P<0.001$ ). Similarly, a greater proportion of patients in the inappropriate use group had predicted 6-mo revascularization risk that was less than the LAR (ratio $<1)$ as compared with the appropriate/uncertain use group (75 [10.9\%] vs. 14 [1.7\%]; $P<0.001$ ).

\section{Men Versus Women}

As shown in Table 2, the means of ${ }^{99 \mathrm{~m}} \mathrm{Tc}$ radioactivity administered and the effective dose received were not statistically different between men and women. In the overall cohort, women had higher LAR and lower benefit-to-radiation risk ratios (Fig. 2). However, among patients with appropriate/uncertain MPI use, the LAR was not statistically different between men and women, as was the probability of MACE-to-LAR ratio, whereas men continued to have higher 6-mo-revascularization-to-LAR ratio. In the inappropriate use group, there was a wider and statistically significant gap between men and women as far as LAR and both benefit-to-radiation risk ratios (Fig. 2).

\section{Monte Carlo Analyses}

After 10,000 sample drawings of stochastic modeling of LAR risk, we calculated expected 7.11 cancer cases per 10,000 patients in the entire cohort $(\mathrm{CI}=1.99-12.23), 6.06$ cases in 10,000 patients in the appropriate/uncertain use group ( $\mathrm{CI}=2.07-10.08)$, and 8.31 cases in 10,000 patients in the inappropriate use group $(\mathrm{CI}=3.21-13.49)$ (Table 2). After 10,000 sample drawings of the underlying distributions, we found higher MACE-to-LAR and 6-mo-revascularization-to-LAR ratios in the appropriate/uncertain than inappropriate group (Table 3).

\section{Sensitivity Analyses}

Repeated analyses among patients without history of cardiovascular disease $(n=1,293)$ yielded results similar to those from the entire cohort. One exception, in the appropriate/uncertain use group, women had an even greater MACE-to-LAR ratio than men (median, 3.7 vs. 2.6; $P=0.009$ ) (Supplemental Table 3). Furthermore, having excluded patients older than $80 \mathrm{y}$ of age, the study findings would not have changed.

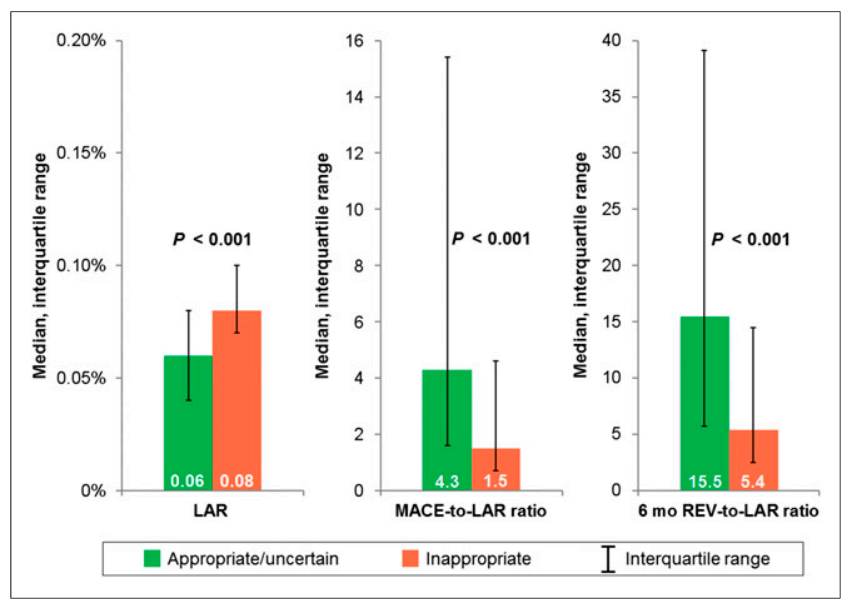

FIGURE 1. LAR of cancer and benefit-to-radiation risk ratios according to appropriateness. Data bars represent median values. Error bars represent interquartile ranges. $P$ values are of the Mann-Whitney test.

\section{DISCUSSION}

To our knowledge, this is the first study to evaluate the impact of appropriate use on the LAR of cancer from radionuclide imaging. We demonstrated that inappropriate MPI use is not only associated with unnecessary cancer risk but also an excess cancer risk as compared with studies performed for an appropriate/uncertain indication. Furthermore, we demonstrated that inappropriate use is detrimental to the benefit-to-radiation risk ratio of SPECT MPI. The impact of appropriate use was most apparent in women; we demonstrated that appropriate/uncertain MPI use closes the sex gap in LAR and benefitto-risk ratio as it relates to prediction of MACE. Our investigation provides experimental evidence for the recommendations of the American Heart Association scientific statement, which emphasizes appropriate patient selection as a mean to enhance radiation safety and maximize the benefit-to-risk ratio of cardiac imaging.

The study subjects underwent a 1-day rest-stress, single-isotope $\left({ }^{99 \mathrm{~m}} \mathrm{Tc}\right)$ protocol using a conventional SPECT camera, a technique implemented in more than $80 \%$ of SPECT MPI studies performed in the United States today (21). The mean effective dose received by the study subjects was $13.6 \mathrm{mSv}$, which is right below the national average of $14.9 \mathrm{mSv}$ reported from the Intersocietal Accreditation Commission (21). A stress-only protocol, using a cadmium zinc telluride $\gamma$-camera, has been shown to reduce the effective dose to as low as $1.4 \mathrm{mSv}$; however, this technique was implemented in less than $1 \%$ of MPI studies performed in U.S. laboratories accredited in 2012 and 2013 (21). Therefore, expanding the use of a stress-only protocol with modern $\gamma$-cameras is a clear opportunity to reducing exposure to ionizing radiation from SPECT MPI. When a stress-only protocol is adopted, the absolute differences in LAR and benefit-to-risk ratios between patients undergoing appropriate versus inappropriate testing and between men and women will diminish; however, relative differences observed in this study are likely to persist. Until radiation-saving protocols are widely implemented, appropriate patient selection will remain the primary mean to reducing exposure to ionizing radiation from MPI.

More recent multimodality 2013 AUC introduced new classification nomenclature: appropriate, may be appropriate, and rarely appropriate, replacing the older terms of appropriate, uncertain, and inappropriate, respectively (22). Although, the new criteria are based on new panel voting, the appropriate use determinations have not changed for most clinical scenarios. Therefore, our study findings are applicable to both 2009 and 2013 AUC.

Cancer risk from the exposure to ionizing radiation is highly controversial. Much of our knowledge of the matter stems from the atomic bomb survivors in Japan (23). Estimates of risk in patients exposed to much lower levels of radiation are based on the linear nonthreshold hypothesis, which assumes that cancer risk increases linearly and accumulatively for any exposure above zero level (11). Discerning whether this hypothesis is true when it comes to lowdose or accumulative exposure from medical imaging is exceedingly difficult because of background natural cancer occurrences and years of latency between the exposure and cancer development. Unless proven to be wrong, the linear nonthreshold hypothesis is the currently accepted principle for estimating the risk from ionizing radiation (11). In recent years, there has been a heightened apprehension of cancer risk as it relates to cardiovascular imaging in general, and MPI in particular (3-7). In fact, MPI is the single medical test with the highest radiation burden and potential cancer risk to the U.S. population $(5,19)$. Berrington de Gonzalez et al. estimated that the 9.1 million MPI studies performed in 2008 may cause 7,400 


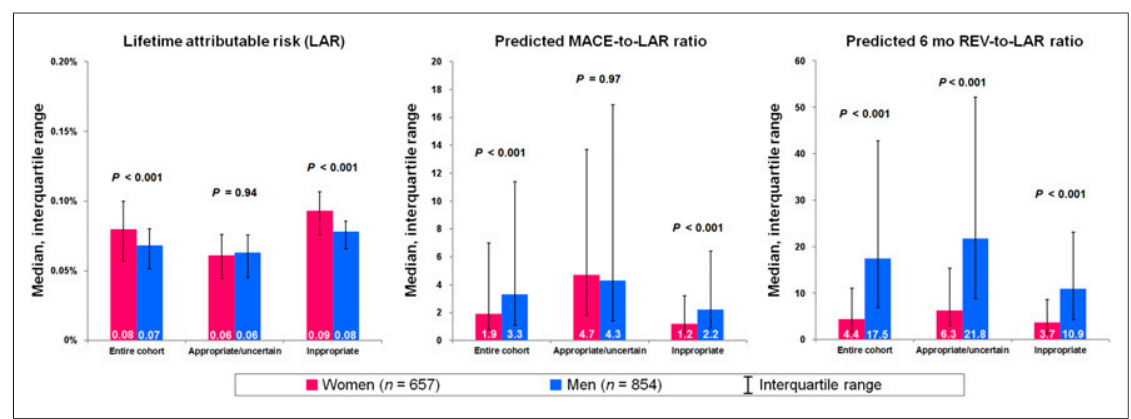

FIGURE 2. LAR of cancer and benefit-to-radiation risk ratios according to appropriateness and sex. Data bars represent median values. Error bars represent interquartile ranges. $P$ values are of the Mann-Whitney test.

(95\% uncertainty interval $=3,300-13,700)$ additional future cancer cases (19). Einstein et al. demonstrated that very high cumulative effective doses are received by patients undergoing MPI, particularly those undergoing repeated testing (6). None of the existing literature addressed cancer risk in the context of predicted clinical benefit or the appropriateness of testing. Furthermore, no study has offered a clinical pathway to select patients for testing according to acceptable or justified exposure level based on the benefit-to-risk ratio. This investigation confirms that the LAR from MPI exposure is greater and the benefit-to-risk ratio is lower among patients who underwent testing for an inappropriate indication. This is not surprising because patients with inappropriate indication are likely to have lower cardiovascular risk, and this patient group is dominated by women and younger individuals who are also at greater cancer risk from ionizing radiation. What was not predictable is demonstrating that appropriate testing closes the sex gap in LAR and benefit-to-LAR ratio from MPI. This is achieved by excluding young low-risk women from unnecessary MPI testing.

This study provides an added dimension for AUC, namely lowering the radiation burden, and offers a framework of thinking about the risk of ionizing radiation by breaking from the absolute cancer risk into benefit-to-risk ratio. Considering that an approximate

TABLE 2

Outcomes According to Sex and Appropriate Use

\begin{tabular}{|c|c|c|c|}
\hline \multirow[b]{2}{*}{ Variable } & \multirow{2}{*}{$\frac{\text { Women }(n=657 ; 43.5 \%)}{\text { Median }}$} & \multirow{2}{*}{$\frac{\text { Men }(n=854 ; 56.5 \%)}{\text { Median }}$} & \multirow[b]{2}{*}{$P^{\star}$} \\
\hline & & & \\
\hline \multicolumn{4}{|l|}{ Radiation exposure and LAR } \\
\hline \multicolumn{4}{|l|}{ Total 99mTc dose (MBq) } \\
\hline Entire cohort & $1,654(1,595-1,721)$ & $1,658(1,598-1,721)$ & 0.97 \\
\hline Appropriate/uncertain & $1,650(1,595-1,717)$ & $1,654(1,598-1,717)$ & 0.91 \\
\hline Inappropriate & $1,658(1,595-1,721)$ & $1,658(1,598-1,783)$ & 0.67 \\
\hline \multicolumn{4}{|l|}{ Effective dose (mSv) } \\
\hline Entire cohort & $13.5(13.1-14.0)$ & $13.5(13.1-14.0)$ & 0.88 \\
\hline Appropriate/uncertain & $13.5(13.0-14.0)$ & $13.5(13.1-14.0)$ & 0.94 \\
\hline Inappropriate & $13.5(13.1-14.0)$ & $13.5(13.0-14.1)$ & 0.98 \\
\hline \multicolumn{4}{|l|}{ LAR of cancer (\%) } \\
\hline Entire cohort & $0.08(0.06-0.10)$ & $0.07(0.05-0.09)$ & $<0.001$ \\
\hline Appropriate/uncertain & $0.06(0.04-0.08)$ & $0.06(0.05-0.08)$ & 0.94 \\
\hline Inappropriate & $0.09(0.07-0.11)$ & $0.08(0.07-0.09)$ & $<0.001$ \\
\hline \multicolumn{4}{|l|}{ Predicted benefit-to-risk ratio } \\
\hline \multicolumn{4}{|l|}{ MACE to LAR ratio } \\
\hline Entire cohort & $1.9(0.8-7.0)$ & $3.3(1.1-11.4)$ & $<0.001$ \\
\hline Appropriate/uncertain & $4.7(1.8-13.7)$ & $4.3(1.4-16.9)$ & 0.97 \\
\hline Inappropriate & $1.2(0.6-3.2)$ & $2.2(0.9-6.4)$ & $<0.001$ \\
\hline \multicolumn{4}{|l|}{ 6-mo-REV-to-LAR ratio } \\
\hline Entire cohort & $4.4(1.7-11.1)$ & $17.5(6.9-42.8)$ & $<0.001$ \\
\hline Appropriate/uncertain & $6.3(2.9-15.4)$ & $21.8(8.8-52.2)$ & $<0.001$ \\
\hline Inappropriate & $3.7(1.2-8.6)$ & $10.9(4.3-23.1)$ & $<0.001$ \\
\hline \multicolumn{4}{|c|}{$\begin{array}{l}\text { *Mann-Whitney test. } \\
\text { REV = revascularization. } \\
\text { Data in parentheses are interquartile ranges. }\end{array}$} \\
\hline
\end{tabular}


TABLE 3

Monte Carlo Models Estimates

\begin{tabular}{|c|c|c|c|}
\hline Variable & Entire cohort & Appropriate/uncertain & Inappropriate \\
\hline \multicolumn{4}{|c|}{ Cancer risk, case/10,000 } \\
\hline All patients & $7.09(2.80-11.38)$ & $6.00(2.56-9.46)$ & $8.31(3.90-12.70)$ \\
\hline Men & $6.43(3.03-9.86)$ & $5.93(1.90-8.71)$ & $7.54(4.67-10.50)$ \\
\hline Women & 7.93 (3.09-12.94) & $6.10(2.41-9.84)$ & $9.09(4.53-13.66)$ \\
\hline \multicolumn{4}{|c|}{ Median MACE-to-LAR ratio } \\
\hline All patients & $3.0(0.2-60.5)$ & $4.6(0.4-90.9)$ & $1.8(0.2-22.7)$ \\
\hline Men & $3.2(0.3-72.3)$ & $4.6(0.4-69.6)$ & $2.5(0.2-23.6)$ \\
\hline Women & $2.2(0.2-36.0)$ & $4.6(0.6-47.1)$ & $1.4(0.2-9.8)$ \\
\hline \multicolumn{4}{|c|}{ Median 6-mo-REV-to-LAR Ratio } \\
\hline All patients & $9.3(1.0-155.2)$ & $14.6(1.7-174.0)$ & $25.6(0.8-76.2)$ \\
\hline Men & $58.4(2.6-195.3)$ & $20.1(2.9-205)$ & $10.1(2.2-98.9)$ \\
\hline Women & $4.3(0.6-63.5)$ & $6.4(1.0-75.9)$ & $3.2(0.7-34.5)$ \\
\hline $\begin{array}{l}\text { REV = revascularizatic } \\
\text { Data in parentheses a }\end{array}$ & & & \\
\hline
\end{tabular}

6 million MPI studies are performed in the United States annually, 1 million inappropriate MPI may lead to an estimated 231 additional cancer cases, above what is expected for appropriate/ uncertain indications. Therefore, implementing the AUC can lead to substantial reduction in radiation burden in the population, further emphasizing the role of AUC as an invaluable quality measure.

The study is limited by basing the calculated probabilities of MACE and 6-mo revascularization on a relatively limited number of events.

\section{CONCLUSION}

On the basis of the linear nonthreshold hypothesis of the biologic effect of ionizing radiation, inappropriate MPI use is associated with not only unnecessary but also an excess estimated LAR of cancer and lower benefit-to-risk ratio. Appropriate MPI use seems to negate the sex gap in cancer risk related to exposure to ionizing radiation from MPI and enhances the benefit-to-risk ratio of MPI among women. The study supports the implementation of AUC for MPI as a key measure to reducing risk from medical radiation in the community.

\section{DISCLOSURE}

The costs of publication of this article were defrayed in part by the payment of page charges. Therefore, and solely to indicate this fact, this article is hereby marked "advertisement" in accordance with 18 USC section 1734 . The original study leading to the formation of the cohort was funded by an investigatorinitiated grant from Astellas Pharma US (Northbrook, IL). The funding source had no input in the study design, execution, data analysis and interpretation, or manuscript preparation and approval. Rami Doukky serves on the Advisory Board for Astellas Pharma and receives research funding from Astellas Pharma. No other potential conflict of interest relevant to this article was reported.

\section{REFERENCES}

1. Doukky R, Frogge N, Balakrishnan G, et al. The prognostic value of cardiac SPECT performed at the primary care physician's office. J Nucl Cardiol. 2013; 20:519-528.

2. Hendel RC, Berman DS, Di Carli MF, et al. ACCF/ASNC/ACR/AHA/ASE/ SCCT/SCMR/SNM 2009 appropriate use criteria for cardiac radionuclide imaging: a report of the American College of Cardiology Foundation Appropriate Use Criteria Task Force, the American Society of Nuclear Cardiology, the American College of Radiology, the American Heart Association, the American Society of Echocardiography, the Society of Cardiovascular Computed Tomography, the Society for Cardiovascular Magnetic Resonance, and the Society of Nuclear Medicine. Endorsed by the American College of Emergency Physicians. J Am Coll Cardiol. 2009;53:2201-2229.

3. Eisenberg MJ, Afilalo J, Lawler PR, Abrahamowicz M, Richard H, Pilote L. Cancer risk related to low-dose ionizing radiation from cardiac imaging in patients after acute myocardial infarction. CMAJ. 2011;183:430-436.

4. Einstein AJ, Henzlova MJ, Rajagopalan S. Estimating risk of cancer associated with radiation exposure from 64-slice computed tomography coronary angiography. JAMA. 2007;298:317-323.

5. Einstein AJ, Moser KW, Thompson RC, Cerqueira MD, Henzlova MJ. Radiation dose to patients from cardiac diagnostic imaging. Circulation. 2007;116:12901305 .

6. Einstein AJ, Weiner SD, Bernheim A, et al. Multiple testing, cumulative radiation dose, and clinical indications in patients undergoing myocardial perfusion imaging. JAMA. 2010;304:2137-2144.

7. Einstein AJ, Tilkemeier P, Fazel R, Rakotoarivelo H, Shaw LJ. Radiation safety in nuclear cardiology-current knowledge and practice: results from the 2011 American Society of Nuclear Cardiology member survey. JAMA Intern Med. 2013;173:1021-1023.

8. Fazel R, Gerber TC, Balter S, et al. Approaches to enhancing radiation safety in cardiovascular imaging: a scientific statement from the American Heart Association. Circulation. 2014;130:1730-1748.

9. Doukky R, Hayes K, Frogge N, et al. Impact of appropriate use on the prognostic value of single-photon emission computed tomography myocardial perfusion imaging. Circulation. 2013;128:1634-1643.

10. Doukky R, Hayes K, Frogge N. Appropriate use criteria for SPECT myocardial perfusion imaging: Are they appropriate for women? J Nucl Cardiol. August 25, 2015 [Epub ahead of print].

11. Committee to Assess Health Risks from Exposure to Low Levels of Ionizing Radiation, Nuclear Radiation Studies Board, Division on Earth Life Studies, National Research Council of the National Academies. Health Risks from Exposure to Low Levels of Ionizing Radiation: BEIR VII Phase 2. Washington, DC: National Academies Press; 2006. 
12. Wilson PW, D'Agostino RB, Levy D, Belanger AM, Silbershatz H, Kannel WB. Prediction of coronary heart disease using risk factor categories. Circulation. 1998;97:1837-1847.

13. Diamond GA, Forrester JS. Analysis of probability as an aid in the clinical diagnosis of coronary-artery disease. N Engl J Med. 1979;300:13501358.

14. Henzlova MJ, Cerqueira MD, Mahmarian JJ, Yao SS. Stress protocols and tracers. J Nucl Cardiol. 2006;13:e80-e90.

15. Doukky R. Pharmacologic stress testing in myocardial perfusion imaging: technical applications. In: Mann A, Heller GV, Hendel RC, eds. Nuclear Cardiology: Technical Applications. New York, NY: McGraw-Hill; 2007: 107-124.

16. Doukky R, Frogge N, Bayissa YA, et al. The prognostic value of transient ischemic dilatation with otherwise normal SPECT myocardial perfusion imaging: a cautionary note in patients with diabetes and coronary artery disease. J Nucl Cardiol. 2013;20:774-784.

17. Mettler FA Jr, Huda W, Yoshizumi TT, Mahesh M. Effective doses in radiology and diagnostic nuclear medicine: a catalog. Radiology. 2008;248:254263.

18. Lantheus. CARDIOLITE kit for the preparation of technetium Tc99m sestamibi for injection [package insert]. Lantheus website. http://www.lantheus.com/PDF/ pi/Cardiolite\%20US\%20PI\%20513121-0710\%207-20-2010.pdf. July 10, 2010. Accessed April 22, 2016.
19. Berrington de Gonzalez A, Kim KP, Smith-Bindman R, McAreavey D. Myocardial perfusion scans: projected population cancer risks from current levels of use in the United States. Circulation. 2010;122:2403-2410.

20. Brenner DJ, Shuryak I, Einstein AJ. Impact of reduced patient life expectancy on potential cancer risks from radiologic imaging. Radiology. 2011;261:193-198.

21. Jerome SD, Tilkemeier PL, Farrell MB, Shaw LJ. Nationwide laboratory adherence to myocardial perfusion imaging radiation dose reduction practices: a report from the Intersocietal Accreditation Commission Data Repository. JACC Cardiovasc Imaging. 2015;8:1170-1176.

22. Ronan G, Wolk MJ, Bailey SR, et al. ACCF/AHA/ASE/ASNC/HFSA/HRS/ SCAI/SCCT/SCMR/STS 2013 multimodality appropriate use criteria for the detection and risk assessment of stable ischemic heart disease: a report of the American College of Cardiology Foundation Appropriate Use Criteria Task Force, American Heart Association, American Society of Echocardiography, American Society of Nuclear Cardiology, Heart Failure Society of America, Heart Rhythm Society, Society for Cardiovascular Angiography and Interventions, Society of Cardiovascular Computed Tomography, Society for Cardiovascular Magnetic Resonance, and Society of Thoracic Surgeons. J Nucl Cardiol. 2014;21:192-220.

23. Preston DL, Shimizu Y, Pierce DA, Suyama A, Mabuchi K. Studies of mortality of atomic bomb survivors: report 13-solid cancer and noncancer disease mortality: 1950-1997. Radiat Res. 2003;160:381-407. 